\title{
TUMOURS AND TUMOUR - LIKE LESIONS OF SCAPULA
}

\author{
Jindra Brtková ${ }^{1}$, Andreas Nidecker ${ }^{2}$, Helena Zídková ${ }^{3}$, Gernot Jundt ${ }^{4}$
}

Charles University in Prague, Faculty of Medicine in Hradec Králové: Department of Radiodiology ${ }^{1}$; Röntgeninstitut Dres. med. PD A. Nidecker, U. F. Benz, E.Barczay, B. Burckhardt, Basle ${ }^{2}$; Charles University in Prague: Department of Radiodiagnostics $^{3}$; Bone Tumour Reference Centre at the Institute of Pathology, Kantonsspital/University Clinics, Basle 4

Summary: A retrospective study of 68 cases of tumours and tumour - like lesions related to the scapula, included in the registry of the Bone Tumour Reference Centre at the Institute of Pathology / University Clinics, Basle, has been carried out. Each case was evaluated for lesion entity, activity and location, age and sex of the patient, and, in 49 files with available radiographic documentation (mostly plain films), for radiologic appearance, with the aim to predict the histologic diagnosis or at least the correct dignity of the lesion. Statistically most frequent were cartilaginous tumours. More than $1 / 3$ of all cases were osteochondromas, which demonstrated mostly a typical appearance. They were encountered predominantly in the first 3 decades in males and were located most often in the body of the scapula. 1/4 of all cases were chondrosarcomas, which were prevailing in the 4th - 7th decades, but were occasionally found at a younger age too. Chondrosarcomas were located mainly at the lateral scapular margin over the inferior angle and in the acromion and coracoid process and their appearance ranged from typical to falsely benign. $1 / 3$ of the cases represented a number of other benign and malignant histological entities.

Key words: Scapula; Tumours; Tumour - like lesions; Radiography

Supported by the Swiss Group of Clinical Cancer Research (SAKK).

\section{Introduction}

The registry of the Bone Tumour Reference Centre at the Institute of Pathology, Kantonsspital/University Clinics, Basle, contains 68 files of tumours or tumour - like lesions of the scapula gathered since 1971. The aim of this retrospective study is to give a review of this rather large series in an infrequently affected bone, to compare the results with similar reports and to seek answers to the following questions: Encountering a lesion in the scapula - what do we have to think of? What incidence of malignancy must we expect? How much can the patient's age and site of involvement help to suggest a diagnosis? How often can we expect a typical radiologic appearance? For diagnosis, can we rely on the plain films or are crosssectional methods essential?

\section{Materials and methods}

A retrospective study of 68 histologically proven cases (68 patients - 43 male and 25 female, in the age of $1-80$ years) was carried out. Each case was evaluated for lesion entity and activity, age and sex of the patient, location of the lesion and, in 49 files with available radiographic documentation, for radiologic appearance. This included the assessment of the typical appearance of the lesion or the ability at least to predict its benign or malignant character.

Only a minority of the files included other imaging modalities - 16 Computed Tomography (CT) studies, 1 Magnetic Resonance Imaging (MRI) study and 2 angiographic studies. The results of evaluating these methods are therefore very limited.

\section{Results}

In terms of frequency, the series was dominated by cartilaginous tumours: 27 osteochondromas, 16 chondrosarcomas and 3 chondromas, which altogether presented $68 \%$ of the patients. These were closely followed by 3 nonHodgkin's lymphomas. The rest of the cases (approximately one third) was evenly distributed among a variety of entities, demonstrated by Tab. 1 .

11 benign and 8 malignant different diagnostic entities were encountered. 42 patients presented benign tumours or tumour - like lesions, 26 patients malignant tumours, the ratio of benign versus malignant thus being $1,9: 1$ respectively. Among the above mentioned most frequent tumours, this ratio is slightly more in favour of malignancy: 1,6 benign v. 1 malignant lesion. In other words in the entire series 
$35 \%$ of the lesions and in the leading group $40 \%$ of the lesions were malignant.

Tab. 1: Tumours and tumour - like lesions related to the scapula; (1) see literature - ref. (11).

\begin{tabular}{|l|c|l|c|}
\hline $\begin{array}{l}\text { Tumours } \\
\text { (lesions) }\end{array}$ & $\begin{array}{c}\text { No. of } \\
\text { cases }\end{array}$ & $\begin{array}{l}\text { Tumours } \\
\text { (lesions) }\end{array}$ & $\begin{array}{c}\text { No. of } \\
\text { cases }\end{array}$ \\
\hline Osteochondroma & 27 & Chondroblastoma & 1 \\
\hline Chondrosarcoma & 16 & Desmoplastic fibroma & 1 \\
\hline Enchondroma & 3 & Metastasis & 1 \\
\hline Non-H. lymphoma & 3 & Osteoblastoma & 1 \\
\hline Aneurysmal bone cyst & 2 & Traumatic dysplasia (1) & 1 \\
\hline Eosinophilic granuloma & 2 & Osteosarcoma & 1 \\
\hline Ewing sarcoma & 2 & Simple bone cyst & 1 \\
\hline Chronic osteomyelitis & 2 & Sec. involvement & \\
\hline Plasmocytoma & 1 & Synovial sarcoma & 1 \\
\hline Round cell sarcoma & 1 & Myositis ossificans & 1 \\
\hline
\end{tabular}

The age of the patients with more frequent lesions is revealed in Tab. 2. The age of the patients presenting rare lesions was in the typical range for each entity, except for 1 case of a simple bone cyst, diagnosed at the age of 43 years, 1 case of a Ewing sarcoma detected at the age of 2 years and 1 patient with a secondary osteosarcoma (following radiotherapy for hemangioendothelioma 4 years earlier) at the age of 59 years. Sex predominance (Tab.2) was observed in osteochondromas (predominantly male patients). NonHodgkin's lymphomas (in our series also affecting predominantly male patients), and enchondromas (affecting with lower significance predominantly female patients) were represented by small numbers of patients. Chondrosarcomas, on the other hand, displayed an almost equal sex ratio.

Tab. 2: The age (No. of cases related to decades of life) and sex of patients in the most frequent entities.

\begin{tabular}{|l|c|c|c|c|c|c|c|c|c|}
\hline & \multicolumn{7}{|c|}{ Age (decades) } & \multirow{2}{*}{ M : F } \\
\hline Tumours & 1 & 2 & 3 & 4 & 5 & 6 & 7 & 8 & \\
\hline Osteochondromas & & & & & & & & & \\
- strongly proliferating & & 6 & & & & & & & \\
- mildly proliferating & & 5 & 4 & 2 & 1 & 1 & & & $20: 7$ \\
- nonproliferating & 2 & 2 & 3 & & 1 & & & & \\
\hline Chondrosarcomas & 1 & 2 & 2 & 1 & 5 & & 2 & 3 & $7: 9$ \\
\hline Enchondromas & & 2 & 1 & & & & & & $1: 2$ \\
\hline Non-Hodgkin's lymphomas & & & & & 1 & 1 & 1 & & $3: 0$ \\
\hline
\end{tabular}

Osteochondromas were located mostly in the body of the scapula - 14 (of these 6 in spina scapulae), 2 were in the superior and 2 in the medial margin, 2 in the inferior and 1 in the superior angle (Fig.1a), with prominence dorsally as well as ventrally, twice in both directions. Chondrosarcomas, on the other hand, were encountered mostly in the lateral scapular margin - 6 (of these 5 near the inferior angle), 6 were in appendices ( 3 in the coracoid process and 3 in the acromion) and only 1 in the body (Fig.1b). 1 chondrosarcoma had destroyed the whole scapula. Enchondromas and
non-Hodgkin's lymphomas were distributed evenly. An aneurysmal bone cyst, Ewing sarcomas, osteomyelitis, a plasmocytoma, a round cell sarcoma, an osteoblastoma, an osteosarcoma, a simple bone cyst and a synovial sarcoma (extending from adjacent soft tissues) were distributed evenly in the upper half of scapula, the rest of the entities were in the lower half.

Out of the 16 radiologically documented osteochondromas, 12 were assessed as typical (Fig. 2 and 3), 3 as benign lesions (mostly widening of a scapular structure by a very broad osteochondroma), 1 was considered equivocal in terms of possible malignization (Fig. 4, malignization was not histologically proved). The 2 osteochondromas still proliferating at the age of 41 and 52 years both revealed a typical apperance.

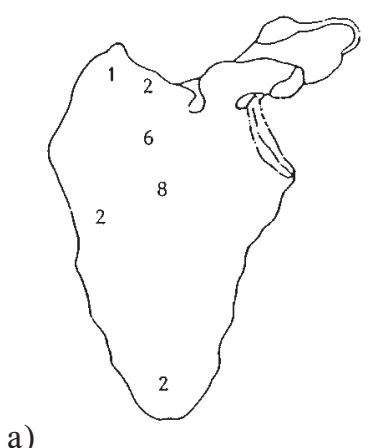

b)

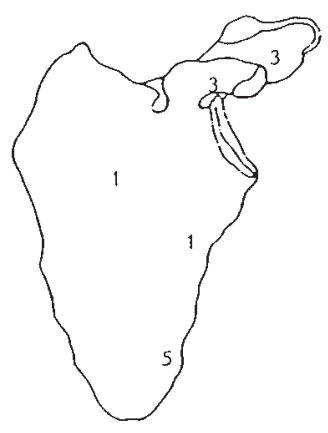

Fig. 1: Localization of osteochondromas (a) and chondrosarcomas (b) in the scapula.

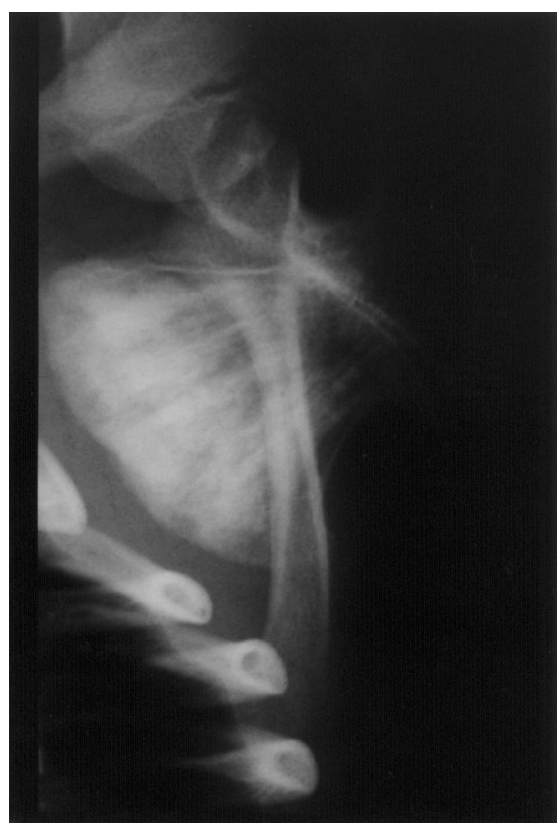

Fig. 2: A typical osteochondroma arising from the anterior surface of the scapula, demonstrating continuous cortical and cancellous bone and massive calcification. 


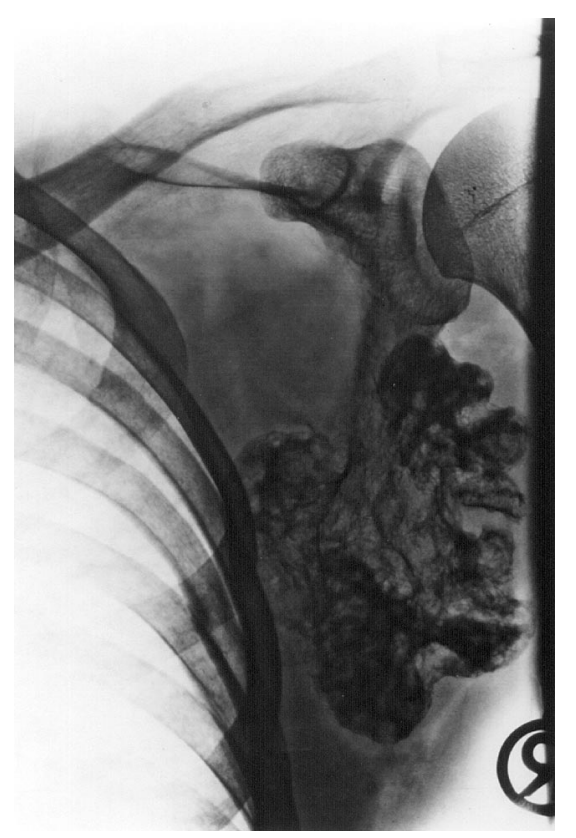

Fig. 3: A typical osteochondroma arising from the inferior angle of the scapula with prominence both ventrally and dorsally, demonstrating a lobulated shape with continous cortical bone and a sharp margin.

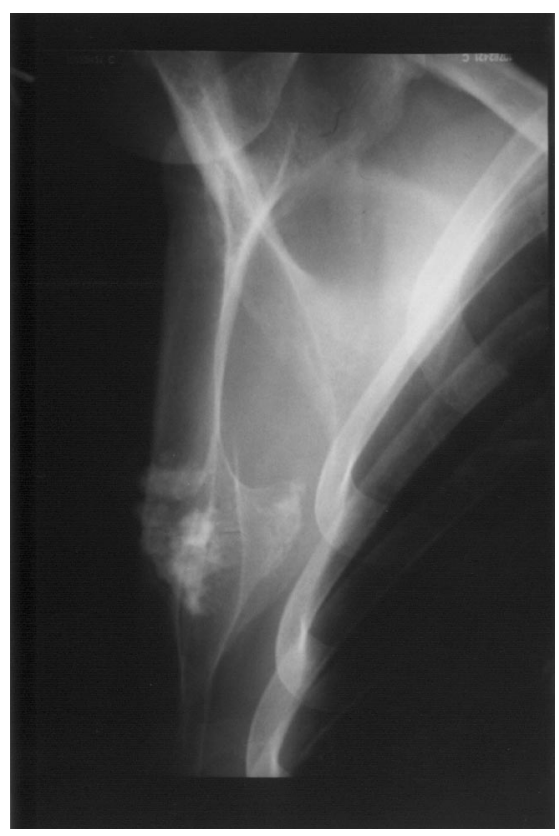

Fig. 4: Osteochondroma located over the inferior angle of the scapula with a typical prominence ventrally, demonstrating smooth continuous cortical and cancellous bone, and a prominence dorsally of equivocal appearance due to superimposed massive unsharp calcifications.

No histological evidence of malignization was found, the cartilage cap was thin, the calcifications were located in the osseous part of the prominence.

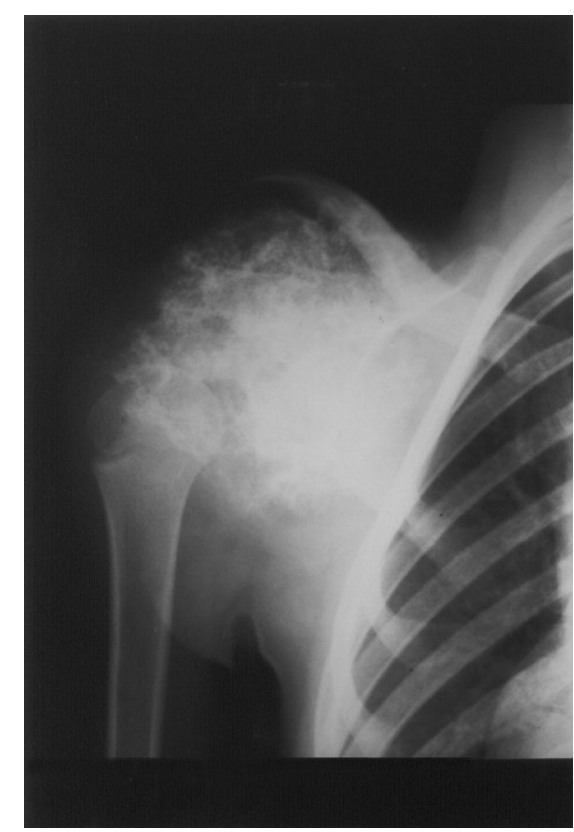

Fig. 5: A typical chondrosarcoma arising most probably from the coracoid process, demonstrating typical calcifcations in rings and semircircles of sharp margins in a large soft tissue mass. The osteolytic component of the tumour is ovelapped by the calcifications. A deviation and a light erosion of the inferior aspect of the lateral part of the clavicula are revealed. The age of the patient ( 6 years old) is uncommon.

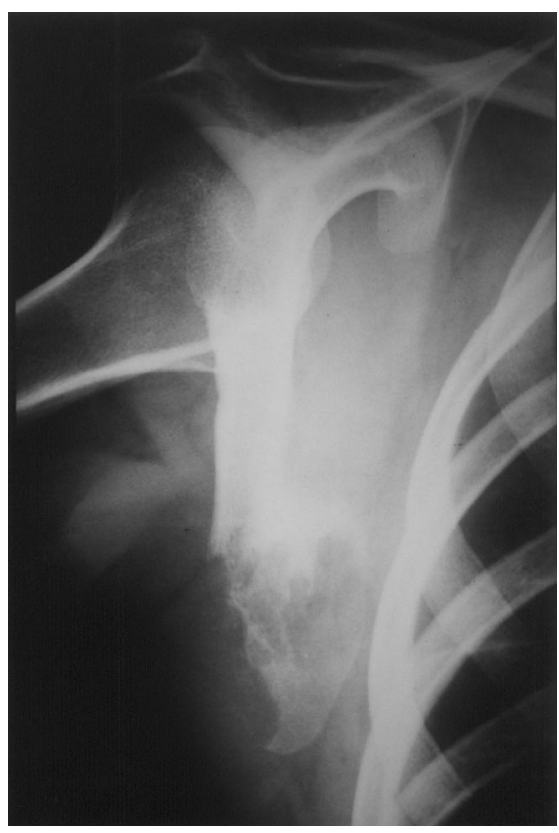

Fig. 6: A chondrosarcoma with a malignant but atypical appearance, demonstrating inhomogenous osteolysis and broad destruction of the lateral cortical bone over the inferior angle and a soft tissue mass, which contains very scarce calcifications. The osteolysis is partly surrounded by an irregular sclerotic rim. 
Out of the 13 radiologically documented chondrosarcomas, 5 were assessed as typical (Fig. 5), 5 as malignant, atypical for a cartilaginous tumour (Fig. 6), 1 was classified as equivocal (Fig. 7), the appearance of 2 chondrosarcomas was falsely judged as benign (Fig. 8).

Out of the 2 radiologically documented enchondromas, 1 was assessed as benign, the other as equivocal, none revealed typical calcifications, partly due to projection.

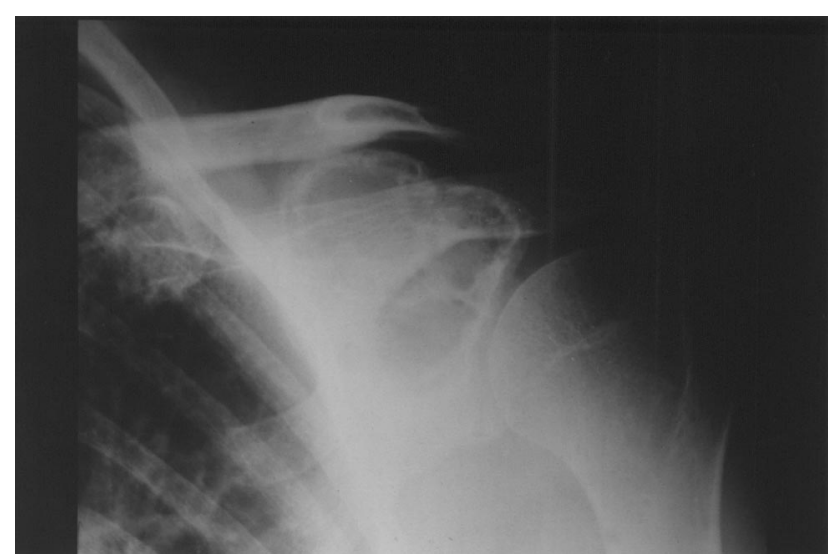

Fig. 7: A chondrosarcoma of an equivocal appearance in the coracoid process, demonstrating a very expansile character without destruction of the cortical bone and with a well defined irregular sclerotic rim. The tumour contains thick septations and very scarce calcifications. No soft tissue mass is revealed, the clavicula demonstrates an unaggressive erosion of its inferior aspect.

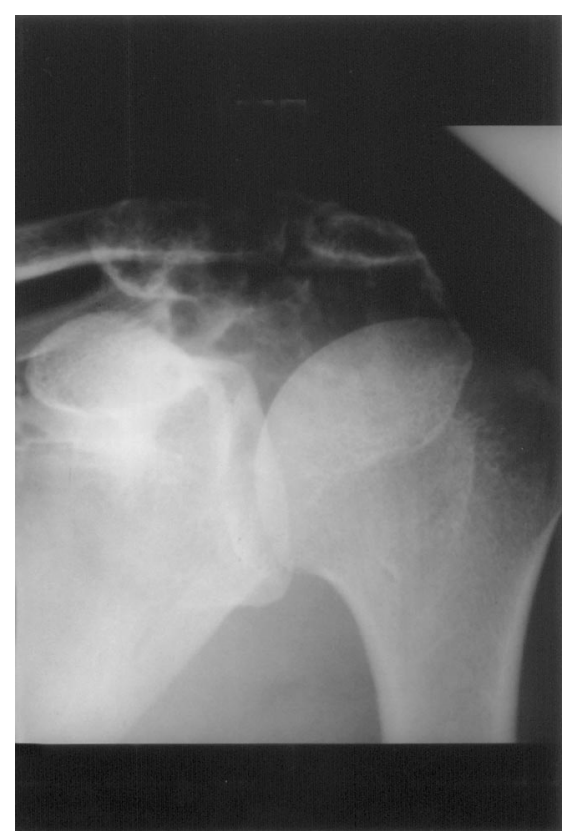

Fig. 8a: A chondrosarcoma with a very expansile falsely benign appearance in the coracoid process. The cortical bone seems to be thin but mostly continuous, only in two short parts of the cortex minimal destruction could be suspected (a).

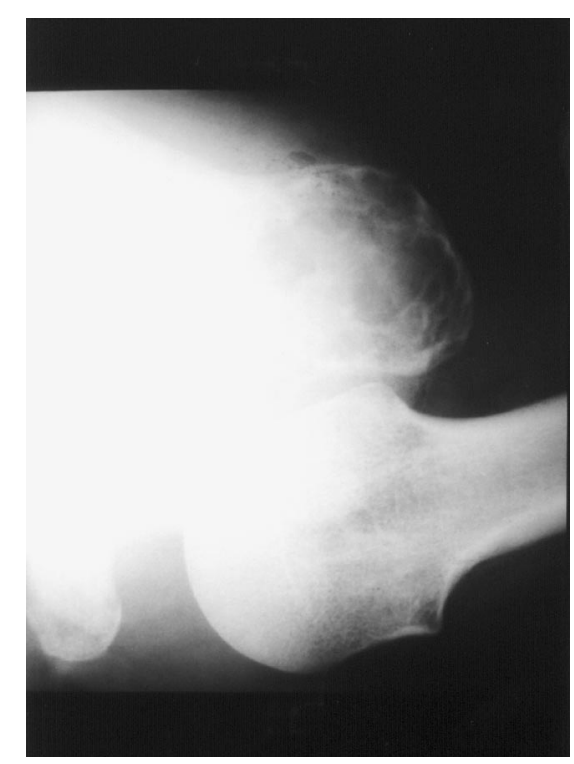

Fig. 8b: The tumour contains thin septations and only in 1 projection (b), a few calcifications could be detected.

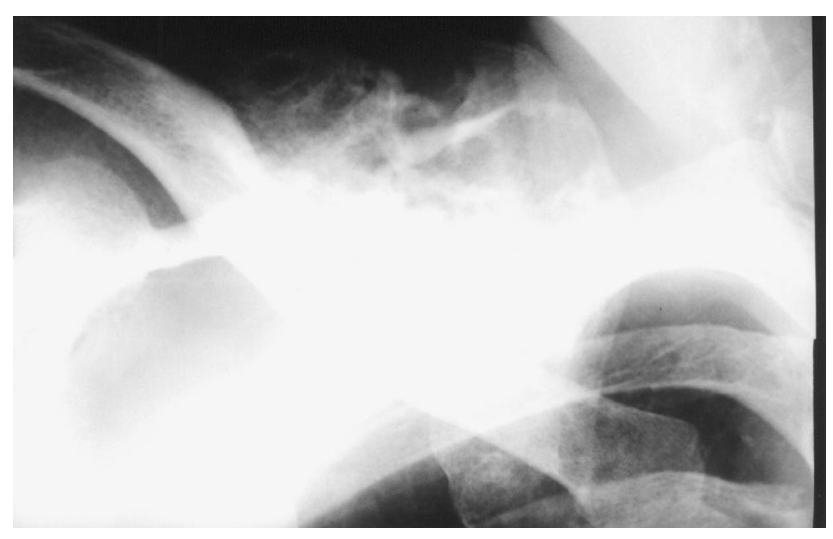

Fig. 9a: A non-Hodgkin's lymphoma of the bone in the medial part of the scapula, demonstrating a very expansile character and thick septations. The plain film arouses no suspicion of cortical destruction.

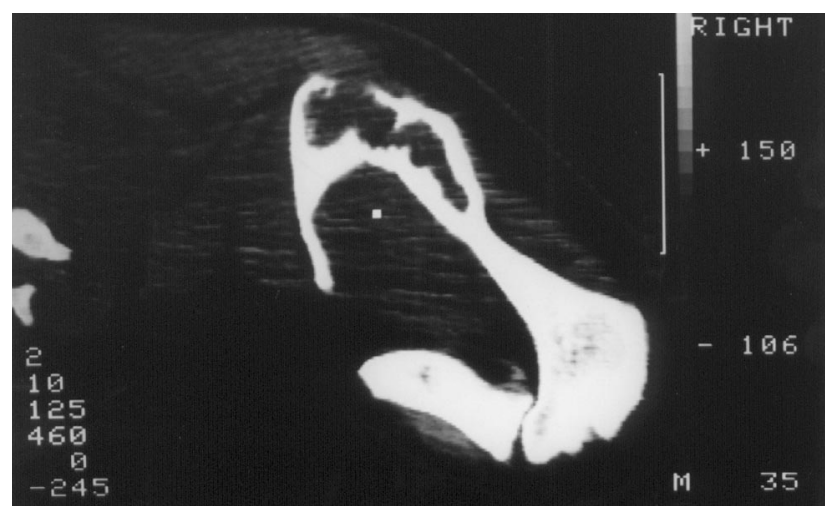

Fig. 9b: The CT scan, on the other hand, shows the destruction of a considerable part of the dorsal cortex without a soft tissue mass. 


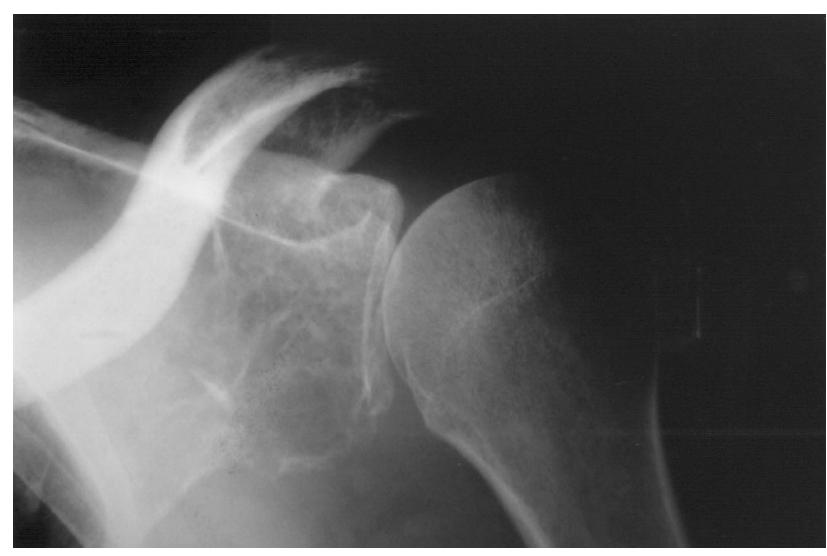

Fig. 10: A plasmocytoma in the inferior region of the neck of the scapula, demonstrating an unsharp geographical osteolysis with a short but definite destruction of the cortical bone in the inferior aspect of the neck.

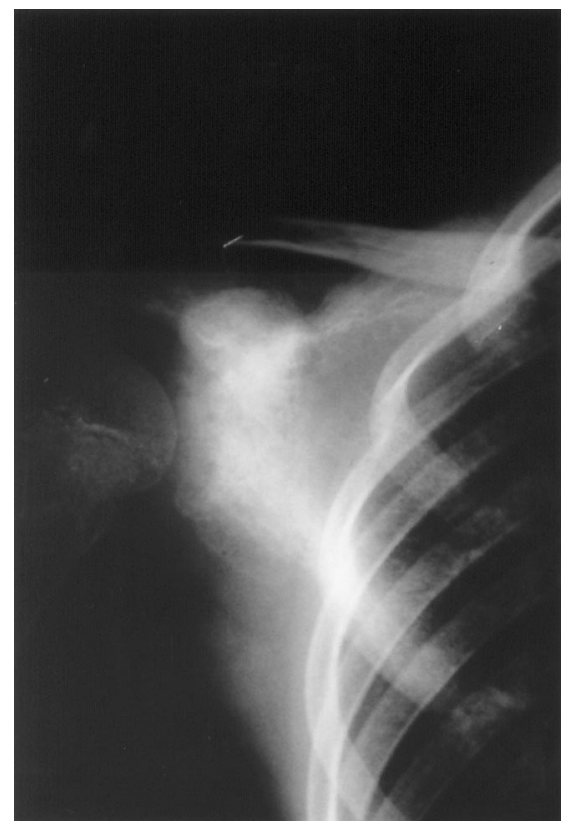

Fig. 11: A Ewing sarcoma demonstrating diffuse sclerotic infiltration of the glenoid, neck, coracoid process and superior and inferior margins of the scapula, extending without sharp margins into the body of the bone. No original cortex is revealed, the involved parts of the scapula are enlarged by periosteal appositions. An unsharp erosion of the medial aspect of the humeral metaphysis is present.

Out of the 3 non-Hodgkin's lymphomas, 1 had the appearance of an equivocal lesion with sharp margins and almost continuous cortical bone on $\mathrm{X}$ - ray, which was later demonstrated by CT as discontinuous (Fig. 9), the other 2 lymphomas appeared malignant - 1 with permeative osteolysis and a pathological fracture, the other - though histologically classified as highly malignant - demonstrated a malignant but only mildly aggressive appearance.

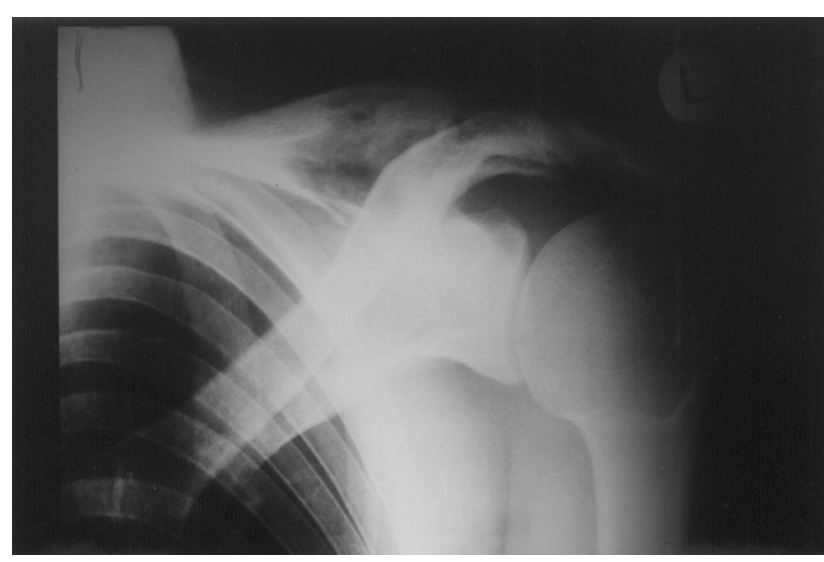

Fig. 12a: Chronic osteomyelitis of the scapula involving the acromion, the spina and extending into the body of the scapula. The lesion demonstrates diffuse inhomogenous osteosclerosis engulfing a zone of irregular partly unsharp osteolysis and extensive periostosis $(a, b)$.

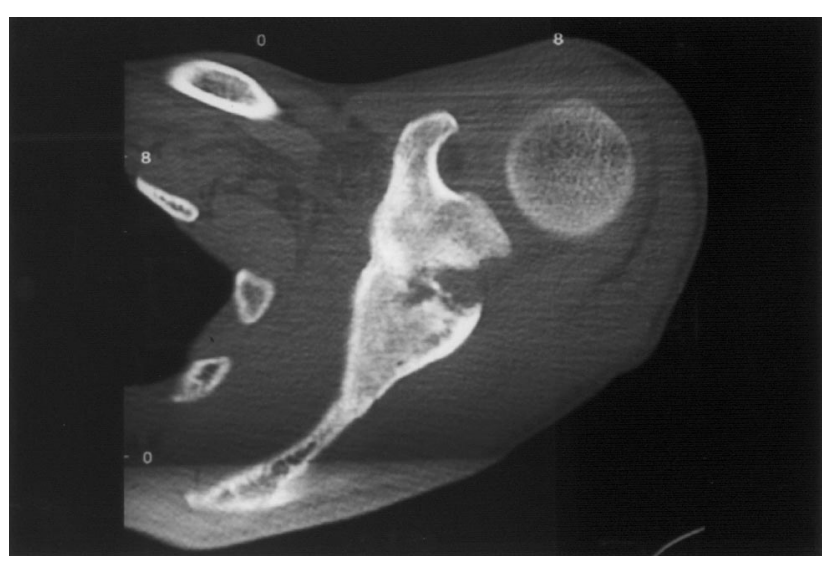

Fig. 12b: The CT scan reveales obscured fat planes in the soft tissues dorsally.

One plasmocytoma (Fig. 10), the 2 Ewing sarcomas - both markedly sclerotic (Fig. 11), one round cell sarcoma and an osteosarcoma all had an unspecific malignant appearance.

The eosinophilic granuloma (Langerhans cell histiocytosis) and the simple bone cyst had a benign but very expansive appearance. The 2 cases of chronic osteomyelitis had in 1 case a typical appearance (Fig. 12), the other looked unspecific benign. The chondroblastoma, apart from its location in the neck extending to the infraspinal fossa, appeared typical. The desmoplastic fibroma appeared unaggressive, although its cortex was very thin and partly discontinuous. Myositis ossificans could not be distinguished from an osteochondroma due to superimposition of a neighbouring bony structure. An osteoblastoma demonstrated a very typical appearance (Fig. 13). The aneurysmal bone cyst, appeared in 1 case as a typical expansile thin walled lesion, in the other case as a highly malignant tumour with a completely dissolved acromion. Very confusing was the 


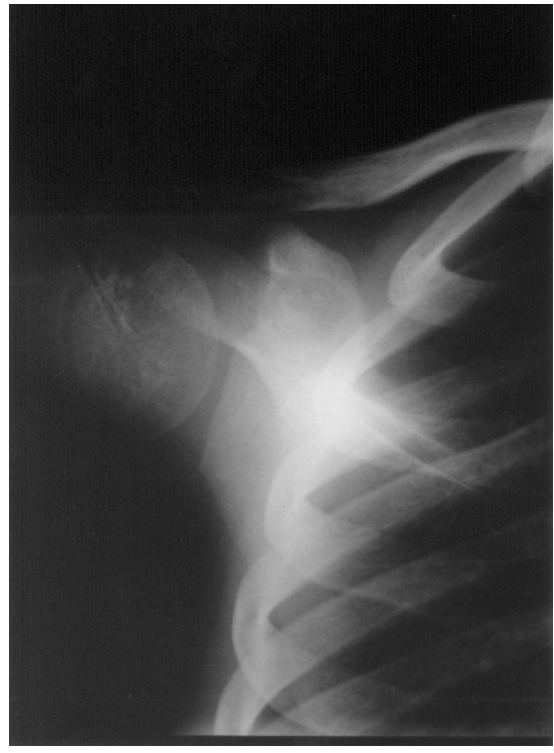

Fig. 13 a,b: A typical osteoblastoma presenting as a round osteolytic lesion in the base of the coracoid process, with sharp margins and a sclerotic rim, containing a calcified nidus.
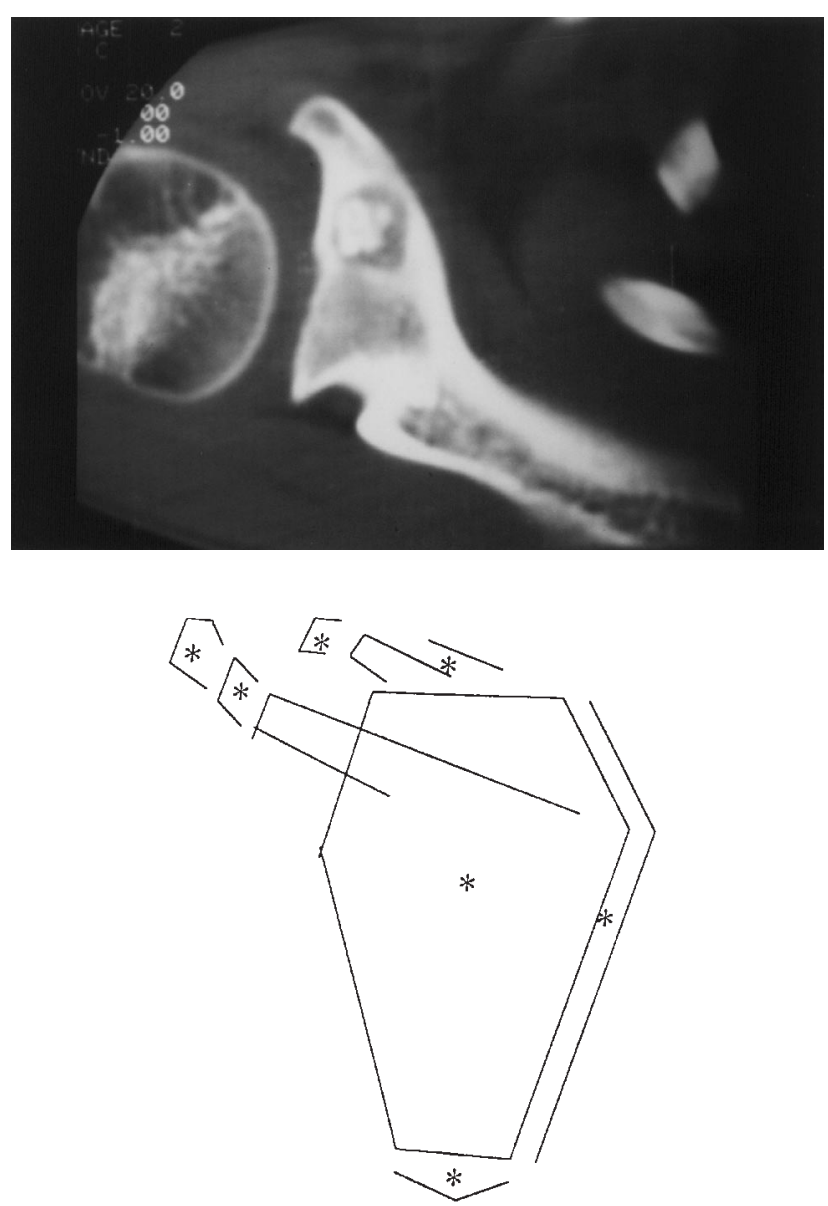

Fig. 14: The 7 ossification centres of the scapula.
Tab. 3a: Radiologic appearance of benign tumours and tumour - like lesions.

\begin{tabular}{|l|c|c|c|c|}
\hline Tumour & \multicolumn{4}{|c|}{ Radiologic appearance (No. of cases) } \\
& Typical & Benign & Equivocal & Malignant \\
\hline Osteochondroma & 12 & 3 & 1 & \\
\hline Endochroma & & 1 & 1 & \\
\hline Aneurysmal bone cyst & 1 & & & 1 \\
\hline Eosinophilic granuloma & & 1 & & \\
\hline Chronic osteomyelitis & 1 & 1 & & \\
\hline Chondroblastoma & & 1 & & \\
\hline Desmoplastic fibroma & & 1 & & \\
\hline Myositis ossificans & & 1 & & \\
\hline Osteoblastoma & 1 & & & \\
\hline Traumatic dysplasia & no & X - ray & & \\
\hline Simple bone cyst & & 1 & & \\
\hline Total & 15 & 10 & 2 & 1 \\
\hline
\end{tabular}

Tab. 3b: Radiologic appearance of malignant tumours.

\begin{tabular}{|c|c|c|c|c|}
\hline \multirow[t]{2}{*}{ Tumour } & \multicolumn{4}{|c|}{ Radiologic appearance (No. of cases) } \\
\hline & Typical & Malignant & |Equivocal| & Benign \\
\hline Chondrosarcoma & 5 & 5 & 1 & 2 \\
\hline $\begin{array}{l}\text { Non-Hodgkin's } \\
\text { lymphoma }\end{array}$ & & 2 & 1 & \\
\hline Ewing sarcoma & & 1 & & \\
\hline Plasmocytoma & & 1 & & \\
\hline Round cell sarcoma & & 1 & & \\
\hline Metastasis & no & X - ray & & \\
\hline Osteosarcoma & & 1 & & \\
\hline Synovial sarcoma & & & & 1 \\
\hline Total & 5 & 11 & 2 & 3 \\
\hline
\end{tabular}

Tab. 3c: Radiologic appearance of all radiologically documented lesions (49 cases).

\begin{tabular}{|l|c|c|c|c|}
\hline Lesions & \multicolumn{4}{|c|}{ Radiologic appearance (No. of cases) } \\
Typical & Typical & $\begin{array}{c}\text { Correct } \\
\text { dignity }\end{array}$ & Equivocal & $\begin{array}{c}\text { Opposite } \\
\text { dignity }\end{array}$ \\
\hline $\begin{array}{l}\text { Benign tumours } \\
\text { and lesions }\end{array}$ & 15 & 10 & 2 & 1 \\
\hline Malignant tumours & 5 & 11 & 2 & 3 \\
\hline Total & 20 & 21 & 4 & 4 \\
\hline
\end{tabular}

appearance of a synovialosarcoma, which resembled a benign intraosseous tumour of the glenoid (Tab. 3a,b,c).

\section{Discussion}

Tumours and tumour - like lesions are rarely located in the scapula. Resnick (18), summarizing several papers, indicates the frequency of scapular location of $0-8 \%$ of all skeletal sites. According to other authors, the scapula is the site of occurrence of $1-3 \%$ of all primary bone tumours. Most of the reports $(12,16,14,21,5,23,13,9)$ therefore review smaller series of scapular lesions, the largest being, to our knowledge, the report of Erlemann and colleagues from the year 1988 (6), who presented 38 cases. This may also be one of the reasons why scapular lesions tend to be a diagnostic problem. 
The scapula develops by enchondral ossification from 7 ossification centres and has a total length of physes exceeding any tubular bone (Fig. 14). The glenoid, the acromion and the coracoid process correspond with the distal end of a tubular bone (2), whereas the medial margin and inferior angle represent the site of highest growth potential (4). It is therefore understandable, that the majority of scapular tumours are of cartilaginous origin, as also found in the reviewed literature $(6,17,22,1,7,19)$. In our series the cartilaginous tumours represented $68 \%$ of all cases - osteochondromas (27), chondrosarcomas (16) and enchondromas (3).

The location of osteochondromas and chondrosascomas was strikingly different and corresponded with the findings of Erlemann and coll. (6). Osteochondromas were mostly located in the body, chondrosarcomas on the other hand mostly at the lateral scapular margin over the inferior angle and in the acromion and coracoid process. All the other groups were evenly distributed without respect to their dignity, with slight prevalence for the upper half of scapula.

The age of the patients at the time of clinical presentation was in osteochondroma mostly in the first 4 decades and in most cases correlated with the activity of the lesion (Tab. 2), fulfilling thus the presumption, that an osteochondroma may grow only to the age of physeal closure (18). The 2 exceptions were patients with proliferating osteochondromas at the age of 41 and 52 years. Both lesions revealed no suspicion of malignant transformation radiologically and histologically.

All 16 chondrosarcomas were differentiated (14: Grade 1, 2: Grade 2). 2 were secondary on previous osteochondroma and enchondroma, 2 were myxoid, 2 were borderline chondrosarcomas / strongly proliferating enchondromas, 9 were central, only 2 were peripheral and 2 were periosteal. The age of the patients was variable, with a prevalence of occurrence after the age of 40 years. One case of a chondrosarcoma was encoutered at the very low age of 6 years and 3 cases at the age of 19, 20 and 21 years (Tab. 2). There was no relation between the age and differentiation of the tumour. The slight prevalence of female patients (male v. female $0,8: 1$ resp.) is in contrast to the male predominance stated in literature (18), but may be atributable to the low number of cases.

In concordance with literature $(20,10,8)$ and almost identical with the data presented by Erlemann and colleagues (6) was the age of the patients. Benign tumours and tumour - like lesions were encountered mostly at a younger age - predominantly in the first 3 decades (with the leading osteochondromas), as opposed to malignant tumours, which revealed slight predominance for the 4 th and 7 th decades (Fig. 15). Uncommon was the occurrence of a Ewing sarcoma at the age of 2 years and the already mentioned chondrosarcoma at the age of 6 years.

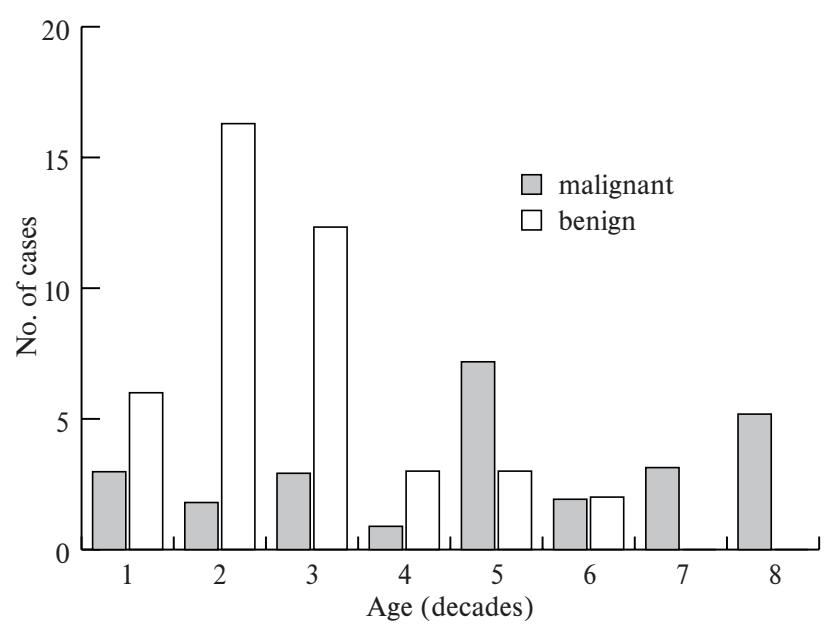

Fig. 15: Relation of age and lesion dignity.

Out of the 68 cases, 49 files were sufficiently documented by X-ray films, only $16 \mathrm{CT}, 1 \mathrm{MRI}$ and 2 angiographic studies were available. The apperance of a lesion on the plain film was considered typical, matching the dignity, equivocal and of falsely opposite dignity as demonstrated by Tab. 3a, b, c, indicating, that a safe diagnosis, i.e. predicting the histological entity or at least the correct dignity was possible in $83 \%$ of the cases, while the correct histological entity itself was predicted in $41 \%$ of the cases. The latter result is somewhat lower than in the very similar study of Erlemann and colleagues (6), who presented $63 \%$ of cases with correct diagnosis while other authors mention the lack of typical features in tumours located in the scapula $(2,7,15,21)$.

In our series the typical appearance was encountered mainly in the group of osteochondromas (12 out of 16). On the other hand in the group of chondrosarcomas a reliable predictive diagnosis was possible only in 5 out of 13 lesions, 5 had a malignant appearance without typical calcifications, 1 was equivocal (a chondrosarcoma secondary on an enchondroma) and 2 displayed even in retrospective benign features. The false negative and equivocal results were mostly caused by a number of unaggressive appearing chondrosarcomas, often demonstrating a pattern of expansile geographic osteolysis with more or less sharp margins and even a sclerotic ring of various thickness, sometimes containing septations. The breakthrough of the cortical bone and typical calcifications were partly not present at all and partly were superimposed over other structures, so that it was or could have been only the CT, that could reveal them. No relation with age, localization or grading was found comparing the aggressive and nonaggressive appearing chondrosarcomas. The 1 available of the 2 periosteal chondrosarcomas had a very atypical appearance resembling a malignant tumour originating from the centre of the bone. The other (documented by an MRI study) had a nonspecific appearance of a soft tissue tumour. 
The remaining groups of infrequent entities, representing $37 \%$ of cases, only rarely revealed a typical X - ray pattern, but mostly allowed a reliable assessment of dignity, except for an aggressive appearing aneurysmal bone cyst and a synovial sarcoma, which resembled a benign intraosseous tumour.

In those 12 cases, where CT and rarely MRI were available in addition to plain films, 9 crosssectional studies didn't contribute to the diagnosis, whereas 3 did, mainly by displaying the otherwise superimposed discontinuity of cortex or small scarce calcifications. As expected, CT and even better MRI revealed the exact topographic extension of the lesion into the adjacent soft tissues.

\section{Conclusion}

In the scapula cartilaginous tumours are statistically the most frequent, composing about $2 / 3$ of all cases.

Among them osteochondromas, representing more than $1 / 3$ of all cases, can usually be diagnosed without greater difficulties (esp. in a child, adolescent or a younger patient, predominantly male, with the lesion localized in the scapular body).

In $1 / 4$ of the cases the heterogenic group of chondrosarcomas should be considered - the peripheral chondrosarcomas with the differential diagnosis of an osteochondroma, the central chondrosarcomas displaying either typical features (ill defined osteolysis, destruction of the cortex, calcifications in rings and points, soft tissue mass), or atypical malignant features without calcifications, or less aggressive equivocal or even benign osteolytic appearance. Even minor signs of malignancy should therefore arouse suspicion. Although the prevailing incidence is from 30 years onward (18), we still have to consider chondrosarcoma in younger patients, even in children. Special concern, including crosssectional studies, must be paid to the localization of a lesion in the lateral scapular margin, acromion and coracoid process (6). In selected cases CT or MRI may be decisive, apart from their unique spacial preoperative information.

In more than $1 / 3$ of cases, the differential diagnosis is broad, including benign and malignant tumours and tumour - like lesions. In children a malignant or even a nonaggressive appearance should suggest a Ewing sarcoma (3), a further differential diagnosis in this age being eosinophilic granuloma (Langerhans cell histiocytosis) (15) and an aneurysmal bone cyst (5). Sharply margined lesions may represent a simple bone cyst (20), an enchondroma, a chondroblastoma (17) and an osteoblastoma - the last 3 possibly containing calcifications. On the other hand nonHodgkin's lymphoma of bone may occur at any age (in our series with a strong male prevalence). A plasmocytoma and a metastasis should be considered in a patient over 40 years of age. Metastatic involvement, according to the literature $(8,10)$ is most common in renal cell carcinoma - our patient with a metastasis of bronchogenic carcinoma was a rarity.
Chronic osteomyelitis should also be considered at this atypical localization, demonstrating patchy destruction, sclerosis and periosteal appositions.

The rarity of envolvement, the broad differential diagnosis and the atypical anatomy make scapula a challenging location for radiologic assessment.

\section{Literature}

1. Abello R, Lomena F, Garcia A et al. Unusual metastatic chondrosarcoma detected with bone scintigraphy. Eur J Nucl Med 1986;12:306-8.

2. Aoki J, Moser RP, Vinh TN. Giant cell tumor of the scapula. Skeletal Radiol 1989; $18: 427-34$

3. Coombs RJ, Zeiss J, McCann K, Phillips E.Case report 360: multifocal Ewing tumor of the skeletal system. Skeletal Radiol 1986;15:254-7.

4. Crocco JA. The Scapula. In: Anatomy Descriptive and Surgical. H.Gray Bounty Books, Crown Publishers Inc,: New York, 1977:143.

5. Damron TA, Brodke DS, Heiner JP, Swan JS, DeSouky S. Case report 803 Gorham's disease (Gorham-Stout syndrome) of scapula. Skeletal Radiol 1993;22:464-7

6. Erlemann R, Davies AM, Edel G, Wuisman P, Peters PE, Grundmann E. Tumoröse Raumforderung der Skapula. Radiologe 1988;28:87-93,

7. Gold RH, Mirra JM. Case report 234: Aneurysmal bone cyst of the left scapula with intramural calcified chondroid. Skeletal Radiol 1983;10:57-60.

8. Guerney H, Larcos G, McKay M, Kefford R, Langlands A. Bone Metastases in Hypernephroma. Frequency of Scapular Involvement. Cancer 1989;64:1429-31.

9. Hermann G, Feldman F, Abdelwahab IF, Klein MJ. Skeletal manifestations of granulocytic sarcoma (chloroma). Skeletal Radiol 1991;20:509-12.

10. Jacobsen KG, Folleras G, Fossa SD. Metastases from renal cell carcinoma to the humerus or the shoulder girdle.Br J Urol 1994;73:124-8.

11. Kandel RA, Pritzker KPH, Bedard YC. Symetrical fibro-osseous dysplasia of rib - post-traumatic dysplasia? Histopathology 1981;5:651-8

12. Kenan S, Lewis MM, Abdelwahab IF, Hermann G, Klein MJ. Case report 652 : Primary intraosseous low grade myxoid sarcoma of the scapula (myxoid liposarcoma). Skeletal Radiol 1991;20:73-5.

13. Kessler S, Mirra JM, Gordon P. Case report 823: Fibro-osseous pseudotumour of the scapula. Skeletal Radiol 1994;23:73-7.

14. Lye DJ, Wepfer JF, Haskell DS. Case report 458: Low grade hemangioendothelioma of the clavicle and acromion. Skeletal Radiol 1988;17:393-401.

15. Moyer RA, Betz RR, Bonakdarpour A. Case report 424: Eosinophilic granuloma of the acroomion. Skeletal Radiol 1987;16:333-5.

16. Pambuccian SE, Horyd ID, Cawte T, Huvos AG. Amyloidoma of bone, a plasma cell/plasmocytoid neoplasm.Report of three cases and review of literature. Am J Surg Pathol 1997; Feb 21(2):179-86.

17. Resendes M, Parker BR, Jones HH, Nagel DA. Case report 663. Unusual chondroblastoma of the Scapula. Skeletal Radiol 1991;20(3):222-5.

18. Resnick D. Bone and Joint Imaging. W.B.Saunders Company:Philadelphia London Toronto Montreal Sydney Tokyo, 1997.

19. Rubenstein DJ, Harkavy L, Glantz L. Case report 518: Periosteal chondroma of scapula. Skeletal Radiol 1989;18:47-9.

20. Ruggieri P, Biagini R, Picci P. Case report 437: Solitary (unicameral, simple) bone cyst of the scapula Skeletal Radiol 1987;16:493-7.

21. Shogry ME, Armstrong P. Case report 630: Reactive bursa formation surrounding an osteochondroma. Skeletal Radiol 1990;19:465-7.

22. Tsuchiya H, Tomita K, Yasutake H, Takagi Y, Ueda Y, Kadoya M. Maffucci's syndrome combined with dedifferentiated chondrosarcoma. Arch Orthop Trauma Surg 1991;110:269-72.

23. Yamato M, Saotome K, Tamai K, Yamaguchi T. Case report 783: Intraosseous ganglion of scapula. Skeletal Radiol 1993;22:227-8.

Submitted February 1999.

Accepted April 1999.

MUDr. Jindra Brtková,

Charles University in Prague,

Faculty of Medicine in Hradec Králové,

Department of Radiology, 50005 Hradec Králové,

Czech Republic.

e-mail: brtkova@fnhk.cz 